\title{
A CHARACTERIZATION OF THE GENERALIZED MEIJER TRANSFORM
}

\author{
E.Y. DEEBA \\ Department of $\Lambda$ pplied Mathematical Sciences \\ University of Hlouston-Downtown \\ llouston, 'Texas 77002 \\ E.L. KOH \\ Department of Mathematics \& Statistics \\ University of Regina \\ Regina, Canada S4S OA2
}

(Received May 8, 1991 and in revised form October, 1991)

\section{ABSTRACT.}

The purpose of the note is to prove a representation theorem for the generalized Meijer transform defined in [2]. In particular, we shall state and prove necessary and sulficient conditions for a function $F(p)$ to be the generalized Meijer transform of a generalized function.

KEYWORDS AND PHRASES. Generalized Meijer transform, generalized function, Bessel differential operator, Representation theorem.

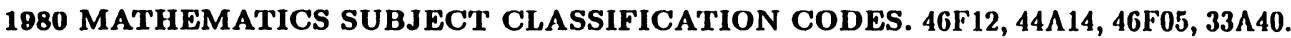

\section{INTRODUCTION.}

The generalized Meijer transform has been defined and studied in [2] and [3] and is given by

$$
\left(\bar{M}_{\mu} f\right)(p)=\frac{2 p}{\Gamma(1-\mu)}<f(t),(p t)^{\mu / 2} K_{\mu}(2 \sqrt{p t})>
$$

where $\mu>-1, K_{\mu}$ is the modified Bessel function of third kind and order $\mu, p$ belongs to a region of the complex plane and $\int$ belongs to the dual $M_{\mu, \gamma}^{\prime}$ of the space $M_{\mu, \gamma}$ defined by

$$
M_{\mu, \gamma}=\left\{\phi \epsilon C^{\infty}(I) \mid \lambda_{\gamma, k}^{\mu}(\phi)<\infty\right\}
$$

and

$$
\lambda_{\gamma, k}^{\prime \prime}(\phi)=\sup _{t \in I}\left|e^{\gamma \sqrt{t}} t^{1-\mu} B_{-\mu}^{k}(\phi(t))\right|, k=0,1,2, \ldots
$$

$\gamma$ being any real number and $B_{-\mu}=t^{\mu} D t^{1-\mu} D\left(D=\frac{d}{d t}\right)$ is the Bessel differential operator. The properties of the space $M_{\mu, \gamma}$ and its dual have been studied in [2]. Furthermore, in [2] the transform (1.1) has been shown to be analytic and an inversion theorem, in the distributional sense, has been established. We note here that if $f(t)$ is locally integrable on $I=(0, \infty)$ and $f(t) e^{-r \sqrt{t}} t^{-1+\mu}$ is absolutely integrable on $I$, then we obtain the classical Meijer transform

$$
\left(M_{\mu} f\right)(p)=\frac{2 p}{\Gamma(1-\mu)} \int_{0}^{\infty} f(t)(p t)^{\mu / 2} K_{\mu}(2 \sqrt{p t}) d t .
$$


In [3], we applied the generalized Meijer transform to a boundary value problem with distributional conditions. To arrive at the solution, it was necessary to use a characterization of the Meijer transform. In this note we shall state and prove necessary and sufficient conditions for a function $F(p)$ to be the generalized Meijer transform of a generalized function $f$ in $M_{\mu, \gamma}^{\prime}$. This will be the content of Section 3 while Section 2 will be devoted to preliminary results and background material.

\section{PRELIMINARIES.}

For the sake of completeness, we shall collect in this section the background material that will be needed in proving the representation theorem.

Throughout we shall denote the interval $(0, \infty)$ by $I$ and $B_{-\mu}^{k}$ the $k$-iterate of the Bessel differential operator. It can be shown that for $B_{-\mu}^{k}$

$$
\begin{gathered}
B_{-\mu}^{k}\left((p t)^{\mu / 2} K_{\mu}(2 \sqrt{p t})\right)=p^{k}(p t)^{\mu / 2} K_{\mu}(2 \sqrt{p t}) \\
B_{-\mu}^{k}\left((p t)^{\mu / 2} I_{\mu}(2 \sqrt{p t})\right)=p^{k}(p t)^{\mu / 2} I_{\mu}(2 \sqrt{p t})
\end{gathered}
$$

where $I_{\mu}$ is the modified Bessel function of the first kind given by

$$
I_{\mu}(2 \sqrt{p t})=\sum_{k=0}^{\infty} \frac{(p t)^{k+\mu / 2}}{k ! \Gamma(k+1+\mu)}, \mu \text { any real number }
$$

and $K_{\mu}$ is the modified Bessel function of the third kind given by

$$
K_{\mu}(2 \sqrt{p t})=\left\{\begin{array}{c}
\frac{\pi}{2 \sin \mu \pi}\left(\sum_{k=0}^{\infty} \frac{(p t)^{k-\mu / 2}}{k !(k+1-\mu)}-\sum_{k=0}^{\infty} \frac{\left(p t t^{\mu / 2+k}\right.}{k !(k+1+\mu)}\right), \mu \text { not integer } \\
\frac{1}{2} \sum_{k=0}^{-\mu-1} \frac{(-1)^{k}(-\mu-k-1) !}{k !}(p t)^{\mu / 2+k}+(-1)^{\mu} \sum_{k=0}^{\infty} \frac{(p t)^{k-\mu / 2}}{k !(-\mu+k) !} \\
\times\left[-\log C(\sqrt{p t})+\frac{1}{2}\left(\sum_{i=1}^{k} \frac{1}{i}+\sum_{i=1}^{-\mu+k} \frac{1}{i}\right)\right], \mu \text { integer. }
\end{array}\right.
$$

$C=e^{\gamma}$ ( $\gamma$ is Euler's constant, Watson [5]). Again, from [5], the asymptotic expansions of $I_{\mu}$ and $K_{\mu}$ are given by

$$
K_{\mu}(2 \sqrt{p t})=\frac{\sqrt{\pi}}{2}(p t)^{\frac{-1}{4}} e^{-2 \sqrt{p t}}\left[1+0\left(|p t|^{\frac{-1}{2}}\right)\right],-\pi<\arg p<\pi
$$

and

$$
I_{\mu}(2 \sqrt{p t})=\left\{\begin{array}{l}
\frac{1}{2 \sqrt{\pi}}(p t)^{\frac{-1}{4}}\left(e^{2 \sqrt{p t}}+i e^{-2 \sqrt{p t}+i \mu \pi}\right)\left[1+0\left(|p t|^{\frac{-1}{2}}\right)\right],-\frac{\pi}{2}<\arg p<\frac{3 \pi}{2} \\
\frac{1}{2 \sqrt{\pi}}(p t)^{\frac{-1}{4}}\left(e^{2 \sqrt{p t}}-i e^{-2 \sqrt{p t}-i \mu \pi}\right)\left[1+0\left(|p t|^{\frac{-1}{2}}\right)\right],-\frac{3 \pi}{2}<\arg p<\frac{\pi}{2}
\end{array}\right.
$$

It was shown in [2] that the space $M_{\mu, \gamma}$ is a complete Fréchet space and that

$$
D_{l}\left[(p t)^{\mu / 2} K_{\mu}(2 \sqrt{p t})\right] \text { and } D_{p}\left[(p t)^{\mu / 2} K_{\mu}(2 \sqrt{p t})\right]
$$

both belong to $M_{\mu, \gamma}$. Further, if $\gamma$ and $\alpha$ are real numbers such that $\gamma<\alpha$, then $M_{\mu, \alpha}$ is a subspace of $M_{\mu, \gamma}$ and the restriction of $f \in M_{\mu, \gamma}^{\prime}$ to $M_{\mu, \alpha}$ is in $M_{\mu, \gamma}^{\prime}$. This implies that there is a real number $\sigma_{f}$, called the abscissa of definition of $f$, such that the restriction of $f$ to $M_{\mu, \gamma}$ is in $M_{\mu, \gamma}^{\prime}$ if $\gamma>\sigma \rho$ and is not in $M_{\mu, \gamma}^{\prime}$ if $\gamma<\sigma_{f}$. the operator $B_{-\mu}^{k}$ and its adjoint are respectively continuous linear operators on $M_{\mu, \gamma}$ and $M_{\mu, \gamma}^{\prime}$. Also, the adjoint $B_{-\mu}^{* k}$ can be shown to be $B_{\mu}^{k}$.

For any $f \in M_{\mu, \gamma}^{\prime}$ and $p \in \Omega_{J}=\left\{p \epsilon C\left|\operatorname{Re} \sqrt{p}>\gamma>\sigma_{f}, p \neq 0,\right| \arg p \mid<\pi\right\}$ the following have been established in [2]:

$$
\text { (i) } \bar{M}_{\mu}\left(B_{-\mu}^{k} f\right)(p)=p^{k}\left(\bar{M}_{\mu} f\right)(p)
$$

which is a basis for an operational calculus of the transform $\bar{M}_{\mu}$

(ii) $\bar{M}_{\mu} f$ is analytic in $\Omega_{f}$ and 


$$
D_{p}\left(\bar{M}_{\mu} f\right)(p)=\frac{2}{\Gamma(1-\mu)}<f(t), D_{p} p(p t)^{\mu / 2} K_{\mu}(2 \sqrt{p t})>
$$

and

(iii) the inversion formula is

$$
f(t)=\lim _{\theta_{1} \rightarrow \pi} \frac{\Gamma(1-\mu)}{2 \pi i} \int_{-\theta_{1}}^{\theta_{1}}\left(\bar{M}_{\mu} f\right)(p) p^{-1}(p t)^{-\mu / 2} I_{\mu}(2 \sqrt{p t}) d p(\theta)
$$

where $p(\theta)=\gamma^{2} / 4 e^{i \theta} \sec ^{2} \theta / 2, \gamma$ is a fixed real number in $\Omega_{f}$ and the limit is to be understood in the sense of convergence in $D^{\prime}(I)$, the dual of the space $D(I)$ of all smooth functions on $I$ whose support is contained in a compact subset $K$ of $I$ equipped with the seni-norms

$$
\rho_{n}(\phi)=\sup _{t \in l}\left|D_{t}^{n}(\phi)\right|
$$

Finally, we remark that if $f(t)$ is locally integrable on $I$ and $f(t) e^{-\gamma \sqrt{t}} t^{-1+\mu}$ is absolutely integrable on $I$, then $f(t)$ generates a regular member $f$ of $M_{\mu, \gamma}^{\prime}$ via

$$
<f, \phi>=\int_{0}^{\infty} f(t) \phi(t) d t .
$$

As noted earlier for such functions the transform in (1.1) reduces to the classical Meijer transform given in (1.2).

A result that will be needed in our proof of the representation theorem is

THEOREM A. [Theorem 4 [1]]. If $R e \mu \geq-\frac{1}{2}, R e \sqrt{p}>\gamma_{0} \geq 0$ and $F(p)$ is analytic and bounded according to $|F(p)|<M|p|^{-q}$ where $q<\frac{3}{2} \operatorname{Re} \mu+2$, then for real $c>\gamma_{0}$ and $\operatorname{Re} \sqrt{p}>c, F(p)=M_{\mu}(f)$ where

\section{MAIN RESULT.}

$$
f(t)=\frac{\Gamma(1+\mu) t^{-\mu / 2}}{2 \pi i} \int_{R \in \sqrt{p}=c} F(p) p^{-1-\mu / 2} I_{\mu}(2 \sqrt{p t}) d p .
$$

In this section we shall give a necessary and sufficient condition for a function $F(p)$ to be the generalized Meijer transform of a function $f$ in $M_{\mu, \gamma}^{\prime}$. As we shall see later in the proof of the necessary part, the real number $\mu$ must be restricted to $-\frac{1}{2} \leq \mu<1$.

Before we state the result, we need the following lemma stated in our centext (see [4], p. 18).

LEMMA 3.1. For any function $f \in M_{\mu, \gamma}^{\prime}$, there exist a positive constant $c$ and a non-negative integer $r$ such that for all $\phi \epsilon M_{\mu, \gamma}$

$$
|<f, \phi>| \leq \cos _{r}(\phi)
$$

where $\rho_{r}=\max _{0 \leq k \leq r}\left\{\lambda_{\gamma, 1}^{\mu}, \lambda_{\gamma, 2}^{\mu}, \ldots, \lambda_{\gamma, k}^{\mu}\right\}$.

THEOREM 3.1. A necessary and sufficient condition for a function $F(p)$ to be the generalized Meijer transform $\bar{M}_{\mu}(f)$ of a generalized function $f$ in $M_{\mu, \gamma}^{\prime}$ is that

(i) there exists a region $\Omega_{f}=\{p \epsilon C|\operatorname{Re} 2 \sqrt{p}>\gamma, p \neq 0,| \arg p \mid<\pi\}$ on which $F(p)$ is analytic,

(ii) $F(p)$ is bounded by a polynomial in $|p|$.

PROOF. Assume that $F(p)=\left(\bar{M}_{\mu f}\right)(p)$ for $f \in M_{\mu, \gamma}^{\prime}$. The analyticity of $F(p)$ follows from Theorem 3.2 of [2]. We only need to prove that $F(p)$ is bounded by a polynomial in $|p|$. Since $(p t)^{\mu / 2} K_{\mu}(2 \sqrt{p t}) \in M_{\mu, \gamma}$, Lemma 3.1 implies that there exist a positive constant $c$ and a non-negative integer $r$ such that 


$$
\begin{aligned}
& |F(p)|=\left|\left(\bar{M}_{\mu} f\right)(p)\right|=\left|\frac{2 p}{\Gamma(1-\mu)}<f(\ell),(p t)^{\mu / 2} K_{\mu}(2 \sqrt{p t})>\right| \\
& \leq c|p| \rho_{r}\left((p t)^{\mu / 2} K_{\mu}(2 \sqrt{p t})\right)
\end{aligned}
$$

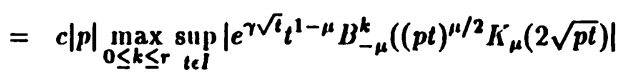

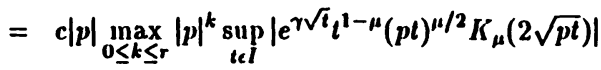

$$
\begin{aligned}
& \leq c|p|^{\gamma+1}
\end{aligned}
$$

by virtue of the series expansion (2.4) and the asymptotic properties $(2.5)$ of $K_{\mu}(2 \sqrt{p t})$. Hence $F(p)$ is bounded by a polynomial in $|p|$.

Assume that $F(p)$ satisfies (i) and (ii) of Theorem 3.1. Let $|F(p)| \leq P_{n}(|p|)$ where $P_{n}(|p|)$ is a polynomial in $|p|$ of degree $n$. Let $q \in R$ be such that $q>\frac{3}{2} R e \mu+2$ and $m$ be an integer such that $m \geq q+n$. Then, for some $M>0,|p|^{-m}|F(p)| \leq M p^{-m}|p|^{n} \leq M|p|^{-q}$. Thus $p^{-m} F(p)$ satisfies the hypothesis of Theorem A stated in Section 2. Therefore, for $\operatorname{Re} \sqrt{p}>c>\gamma_{0}$,

$$
p^{-m} F(p)=M_{\mu}(g)(p)=\frac{2 p}{\Gamma(1+\mu)} \int_{0}^{\infty}(p t)^{\mu / 2} K_{\mu}(2 \sqrt{p t}) g(t) d t
$$

where

$$
g(t)=\frac{\Gamma(1+\mu) t^{-\mu / 2}}{2 \pi i} \int_{R \in \sqrt{p}=c} p^{-m} F(p) p^{-1-\mu / 2} I_{\mu}(2 \sqrt{p t}) d p .
$$

We will show next that $e^{-\gamma \sqrt{t}} t^{-1+\mu} g(t)$ is absolutely integrable on $I$ and conclude from $(2.10)$ that $g(t)$ generates a regular member $g$ of $M_{\mu, \gamma}^{\prime}$.

We consider two cases

(i) for $|p t| \leq 1,0<t<\infty,(2.3)$ implies that

$$
\left|e^{-\gamma \sqrt{t}}(p t)^{-1+\mu / 2} I_{\mu}(2 \sqrt{p t})\right| \leq M e^{-\gamma \sqrt{t}}|p t|^{-1+\mu}
$$

which is integrable on $I$ for $R e \mu>0$.

(ii) for $|p t| \geq 1, \operatorname{Re} \sqrt{p}=c, \operatorname{Re} \mu<1,(2.6)$ implies that

$$
\left|e^{-\gamma \sqrt{t}}(p t)^{-1+\mu / 2} I_{\mu}(2 \sqrt{p t})\right| \leq \frac{1}{2 \sqrt{\pi}}\left|e^{(-\gamma+2 \sqrt{p}) \sqrt{t}}\right||p t|^{\mu / 2-5 / 4}
$$

which is of order $e^{-\alpha \sqrt{t}} t^{-1 / 2}(\alpha>0)$. Thus

$$
e^{-\gamma \sqrt{t}}(p t)^{\mu / 2} I_{\mu}(2 \sqrt{p t}) \text { is absolutely integrable on } 0 \leq t<\infty .
$$

That is,

$$
\int_{0}^{\infty}\left|e^{-\gamma \sqrt{t}}(p t)^{\mu / 2-1} I_{\mu}(2 \sqrt{p t})\right| d t \leq c_{\mu}|p|^{R e \mu}
$$

where $c_{\mu}$ is sufficiently large depending upon $\mu$. To show that $g(t) e^{-r \sqrt{i} t-1+m}$ is absolutely integrable, we invoke Fubini's theorem and the fact

$$
\int_{\operatorname{Re} \sqrt{p}=c>r \geq 0} p^{-m-\mu+\mu} F(p) d p
$$

is finite because $|p|^{-m} F(p) \leq|p|^{-m} P_{n}(|p|) \leq M|p|^{q}$ since $q>\frac{3}{2} R e \mu+2,-q<-(2+\epsilon)$ for our choice of $0<\operatorname{Re} \mu<1$. Thus $(3.2)$ is at least quadratically decreasing for large $p$ along $\operatorname{Re} \sqrt{p}=c$ in both directions. 
We have shown that $g(t) e^{-\gamma \sqrt{t} t^{-1+\mu}}$ is absolutely integrable on $0<t<\infty$. Thus $g(t)$ generates a regular number in $M_{\mu, \gamma}^{\prime}$ for $0<R e \mu<1$. Thus the function $p^{-m} F(p)$ is a generalized Meijer transform whose region of definition is $\{p \mid \operatorname{Re} \sqrt{p}>c>\gamma \geq 0\}$.

We finally note that to find $f \in M_{\mu, \gamma}^{\prime}$ explicitly, we set $f=B_{\mu}^{m} g$. Then $\bar{M}_{\mu} f=\bar{M}_{\mu}\left(B_{\mu}^{m} g\right)=p^{m} \bar{M}_{\mu}(g)=$ $F(p)$.

\section{References}

[1] Conlan, J. and E.L. Koh, On the Meijer transformation, Internat. J. Math and Math. Sci., Vol. 1 (1978), 145-159.

[2] Koh, E.L., E.Y. Deeba, and M.A. Ali, The Meijer Transformation of Generalized Functions, Internat. J. Math and Math. Sciences, Vol. 16, No. 2 (1987), 267-286.

[3] Koh, E.L., E.Y. Deeba, and M.A. Ali, On the generalized Meijer transformation, Generalized Furictions, Convergence Structure and Their Application, Plenum Press, New York, 1988 (pages 219-226).

[4] Zemanian, A.II., Generalized Integral Transformations, Inter-Science, New York, 1968.

[5] Watson, G.N., A Treatise on the Theory of Bessel Functions, Univ. Press, Cambridge, 1966. 


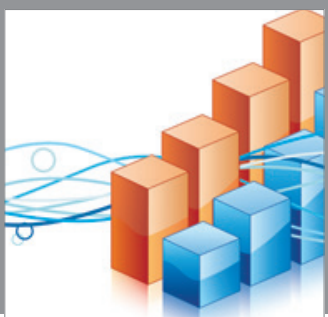

Advances in

Operations Research

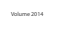

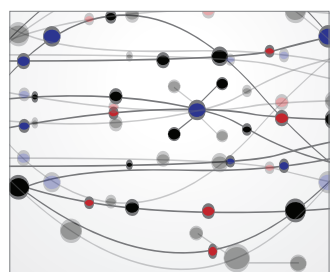

\section{The Scientific} World Journal
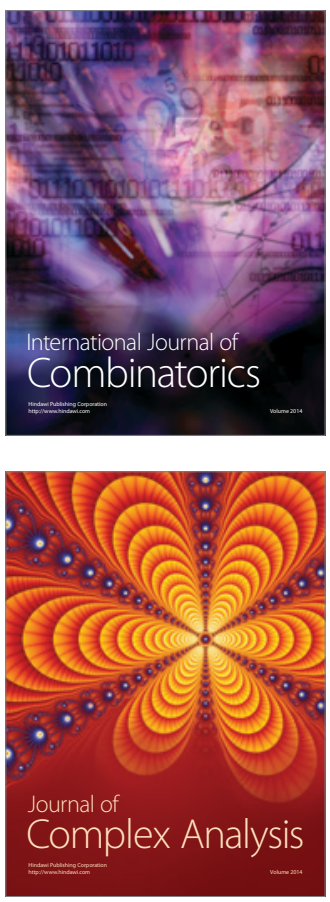

International Journal of

Mathematics and

Mathematical

Sciences
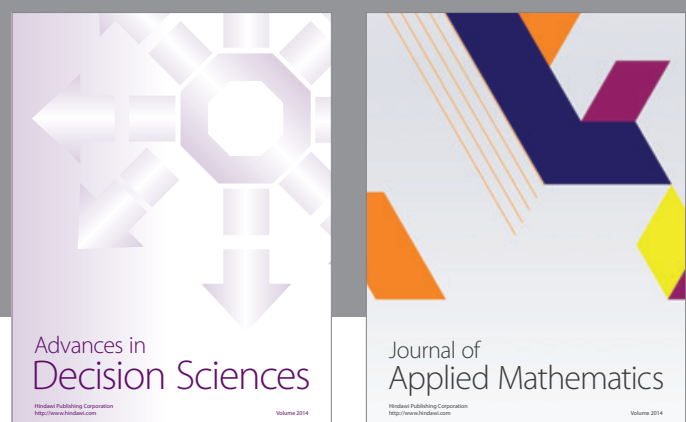

Journal of

Applied Mathematics
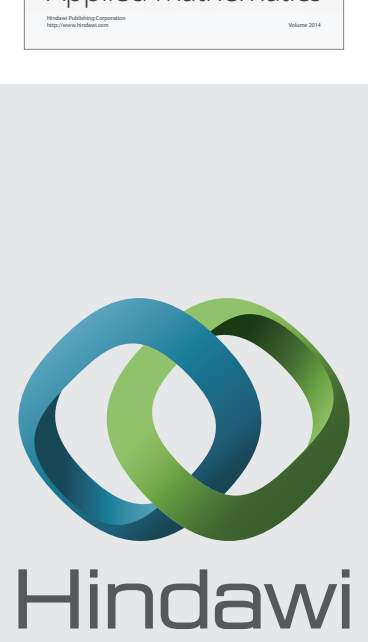

Submit your manuscripts at http://www.hindawi.com
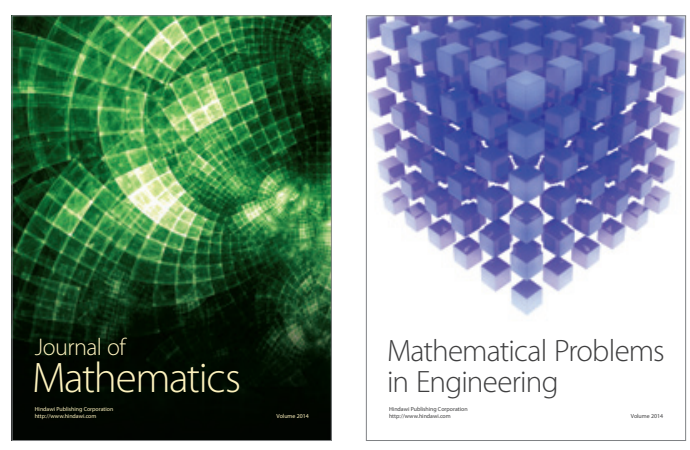

Mathematical Problems in Engineering
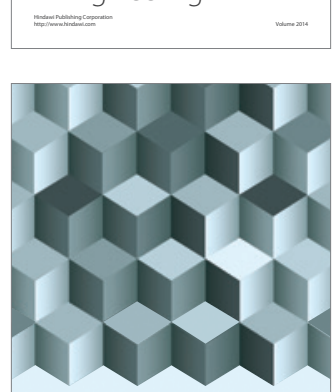

Journal of

Function Spaces
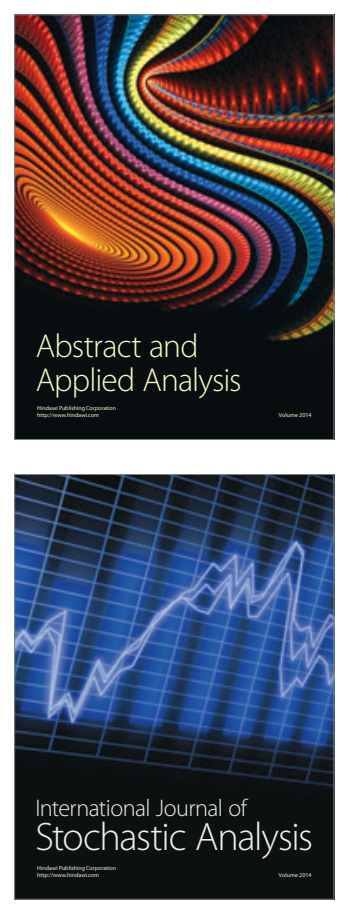

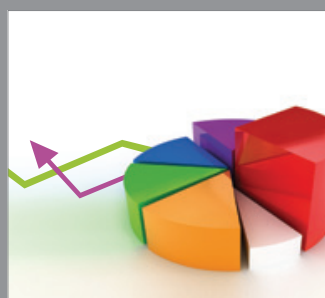

ournal of

Probability and Statistics

Promensencen
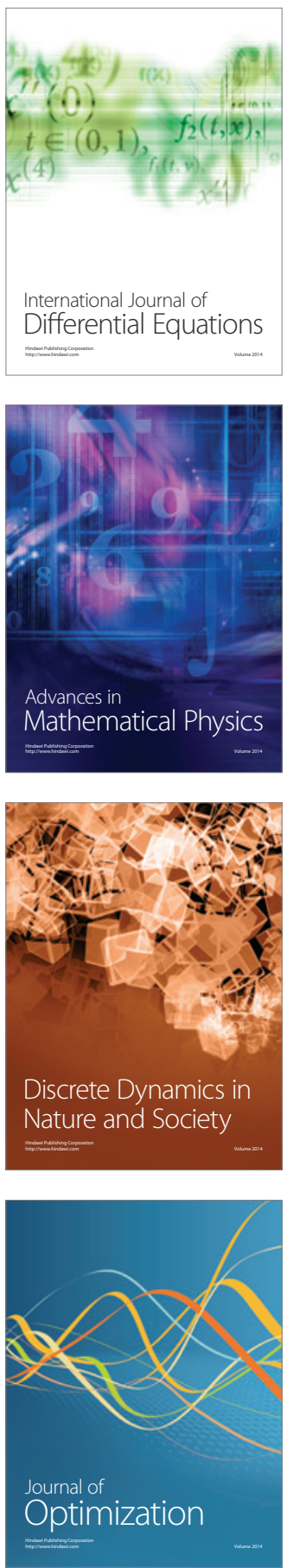